\title{
Erratum to: Trend of determinants of birth interval dynamics in Bangladesh
}

Jahidur Rahman Khan ${ }^{1 *}$, Wasimul Bari ${ }^{2}$ and A. H. M. Mahbub Latif ${ }^{1,3}$

\section{Erratum}

During the processing of this article [1], the following typographical error occurred on page 3: , $u i$ was incorrectly introduced:

1. inside the exponent in the definition of $S_{i j}\left(t \mid X_{i j}, u_{i}\right)$ following Eq. 3; this should read:

$$
\begin{aligned}
S_{i j}\left(t \mid X_{i j}, u_{i}\right) & =\exp \left\{-\int_{0}^{t} h_{i j}\left(x \mid X_{i j}, u_{i}\right) d x\right\} \\
& =\exp \left(-H_{\mathrm{o}}(t) u_{i} \beta^{\prime} X_{i j}\right)
\end{aligned}
$$

2. inside the exponent in Eq. 4; this should read:

$$
\begin{aligned}
L_{m, i}(\phi, \theta, \beta)= & \prod_{j=1}^{n_{i}} \int_{0}^{\infty}\left[h_{0}\left(t_{i j}\right) u_{i} \exp \left(\beta^{\prime} X_{i j}\right)\right]^{\delta_{i j}} \\
& \times S_{i j}\left(t_{i j} \mid X_{i j},, u_{i}\right) g\left(u_{i} ; \theta\right) d u_{i}
\end{aligned}
$$

3. Twice in Eq. 5; this should read:

$$
\begin{aligned}
l_{m}(\phi, \theta, \beta)= & \sum_{i=1}^{K}\left[\log \Gamma\left\{(1 / \theta)+d_{i}\right\}\right. \\
& +\sum_{j=1}^{n_{i}} \delta_{i j}\left(\beta^{\prime} X_{i j}+\log \left(\gamma t_{i j}^{\gamma-1} / \alpha^{\gamma}\right)\right)-\log \Gamma(1 / \theta) \\
& +d_{i} \log \theta-\left\{(1 / \theta)+d_{i}\right\} \\
& \left.\times \log \left(1+\theta \sum_{j=1}^{n_{i}}\left(t_{i j} / \alpha\right)^{\gamma} \exp \left(\beta^{\prime} X_{i j}\right)\right)\right]
\end{aligned}
$$

\section{Author details}

${ }^{1}$ Institute of Statistical Research and Training (ISRT), University of Dhaka, Dhaka 1000, Bangladesh. ${ }^{2}$ Department of Statistics, Biostatistics \& Informatics, University of Dhaka, Dhaka 1000, Bangladesh. ${ }^{3}$ Center for Clinical Epidemiology, St. Luke's International University, 3-6-2 Tsukiji, Chuo-ku, 104-0045 Tokyo, Japan.

Received: 7 October 2016 Accepted: 7 October 2016

Published online: 17 October 2016

\section{References}

1. Khan JR et al. Trend of determinants of birth interval dynamics in Bangladesh. BMC Public Health. 2016;16:934. doi:10.1186/s12889-016-3577-9.

* Correspondence: jkhan@isrt.ac.bd

${ }^{1}$ Institute of Statistical Research and Training (ISRT), University of Dhaka,

Dhaka 1000, Bangladesh

Full list of author information is available at the end of the article 